\title{
Comparative Study of the Influence of Three-Dimensional Versus Two-Dimensional Urological Laparoscopy on Surgeons' Surgical Performance and Ergonomics: A Systematic Review and Meta-Analysis
}

\author{
Francisco Miguel Sánchez-Margallo, PhD,1' David Durán Rey, MS,1 Álvaro Serrano Pascual, MD,² \\ Julio A. Mayol Martínez, MD, ${ }^{3}$ and Juan A. Sánchez-Margallo, PhD ${ }^{1}$
}

\begin{abstract}
Objective: The objective of this study is to compare the use of three-dimensional (3D) vision systems with traditional two-dimensional systems in laparoscopic urological surgery, analyzing the benefits, limitations, and impact of introducing this medical technology with regard to surgical performance and the surgeon's ergonomics. Methods: A systematic review with a structured bibliographic search was conducted in the electronic libraries (PubMed and EMBASE) until August 2019 and with no language restrictions. Studies on 3D visualization technology in laparoscopic urologic surgery, randomized controlled trials, and observational comparative studies were included. Relevant data were extracted and analyzed.

Results: A total of 25 articles were obtained, of which 4 were clinical studies with patients, 2 studies were carried out in experimental animal models, and the remaining 19 were conducted in simulated environments. Regarding the European training program in basic laparoscopic urological skills, the results showed no significant differences in execution time using either imaging system. Three-dimensional vision led to a significant reduction in surgery time in pyeloplasty and radical nephrectomy. In addition, there was a reported decrease in blood loss in adrenalectomy, nephron-sparing nephrectomy, radical nephrectomy, simple nephrectomy, and pyeloplasty using 3D vision. Regarding ergonomics, the studies generally described no differences in side effects (headache, nausea, eye strain) when comparing the two types of visualization systems. Surgeons reported reduced workloads and stress with 3D vision than with traditional laparoscopy.

Conclusions: Three-dimensional laparoscopic systems essentially advance surgical performance in lessexperienced laparoscopic surgeons. Three-dimensional laparoscopy leads to improvements in surgery time, which is important for specific surgical procedures involving intracorporeal ligatures and sutures. The results achieved on the surgeons' ergonomics showed better depth perception and decreased stress and workloads during 3D vision with no differences in potential side effects.
\end{abstract}

Keywords: three-dimensional laparoscopy, three-dimensional vision, three-dimensional imaging, urological surgery, laparoscopic urological surgery, meta-analysis

\section{Introduction}

M INIMALLY INVASIVE SURGERY (MIS) has signaled an undeniable revolution in the field of surgery over the last 30 years, progressively replacing open surgical procedures due, in part, to technological developments such as advanced imaging systems and the introduction of surgical robotics, as well as acceptance by medical consensus of different laparoscopic techniques. ${ }^{1-3}$ This approach has been applied to numerous surgical procedures, and the general belief is that it will continue to spread in the coming years. ${ }^{4}$

The scope of laparoscopic surgery is largely due to the clear benefits it brings to patients, causing less surgical damage, fewer complications and pain, and a faster recovery compared with open surgery. ${ }^{5,6}$ However, this type of approach also entails some limitations for the surgeon due to the

\footnotetext{
${ }^{1}$ Jesús Usón Minimally Invasive Surgery Centre, Cáceres, Spain.

Departments of ${ }^{2}$ Urology and ${ }^{3}$ Surgery, Hospital Universitario Clínico San Carlos, Madrid, Spain.
} 
need to manipulate the instruments and devices at a distance using a two-dimensional (2D) image displayed through screens or monitors. Therefore, adequate training is mandatory before performing this type of surgical technique in a real surgical scenario.

The miniaturization of imaging systems and the current development of high-definition (HD and 4K) and threedimensional (3D) video technologies provide a high-quality surgical vision similar to that offered by open surgery. ${ }^{8,9}$ These innovative imaging technologies enable surgeons to have a shorter learning curve and greater surgical precision. ${ }^{10}$

In the case of 3D vision technology, which has not yet been universally implemented, except in robotic laparoscopic surgery, the general feeling has been that it improves surgeon performance and reduces surgery time compared with classic 2D laparoscopy and that high-definition imaging can also provide benefits for optimal clinical results. ${ }^{10}$ Therefore, in this work, we carry out a systematic review of the scientific literature with the aim of evaluating the effectiveness and safety of 3D laparoscopic vision, comparing the results with traditional 2D vision and paying special attention to surgical procedures in the urology specialty. This study will seek to determine the benefits, limitations, and impact of introducing this medical technology with regard to surgical performance and surgeon ergonomics.

\section{Materials and Methods}

\section{Search strategy}

A structured bibliographic search was conducted in the PubMed and EMBASE electronic libraries. We used the key terms "three-dimensional imaging," "3D," "Laparoscopy”, "Urology," "Urological Surgery," "Urologic Surgical Procedures," "Performance," and "Ergonomics" in appropriate combinations to identify relevant studies published up to August 2019 and with no language restrictions (see "Appendix A1" for the full search strategy). Studies on $3 \mathrm{D}$ visualization technology in laparoscopic urological surgery, randomized controlled trials, and observational comparative studies were also included.

\section{Selection of articles}

Articles that met any of the following criteria were excluded: case reports, retrospective observational comparative studies, studies that addressed only 3D laparoscopy without a comparison to $2 \mathrm{D}$ systems, conference abstracts, or articles published in a language other than English. A flowchart with the different phases of the systematic review was designed according to the recommendations of the PRISMA declaration. ${ }^{11}$

\section{Data extraction}

In each article, general information such as population and detailed information about the task or surgical procedure and experience level of the surgeons were gathered. In addition, relevant data were extracted and analyzed, including the number of surgeons and/or patients, surgery time, estimated blood loss, complications and other assessment criteria for surgical performance, ergonomics and evaluation methods, including statistical significance where appropriate.

\section{Data analyses}

A meta-analysis was carried out to compare the surgical performance using 3D and $2 \mathrm{D}$ vision by analyzing the execution time and errors in laparoscopic training tasks, as well as surgical time in urological procedures. The results are presented as forest plots with $95 \%$ confidence intervals (CIs). A random-effects model was used, and the heterogeneity of the studies was assessed using the I 2 statistic. Summary results are presented as diamonds whose extremities show the CI for the summary estimate. When individual studies were too dissimilar to determine an average estimation of the effect (meta-analytic), summary values were omitted. All statistical analyses were carried out using $\mathrm{R}$ version 3.6.1 (R Foundation for Statistical Computing, Vienna, Austria).

\section{Results}

The bibliographic search in the PubMed electronic library generated a total of 114 articles, of which 15 were not written in English and 72 corresponded to an incorrect subject. Moreover, we also found the presentation of a clinical case and 2 reviews, so the search was narrowed to 24 articles. Regarding the EMBASE search, once the established inclusion and exclusion criteria were applied, 199 articles were generated, of which 74 were duplicated in the PubMed search. Of the remaining 125 articles, one article was written in a language other than English, 35 were conference abstracts, and 88 corresponded to incorrect subject matter, reducing the search to 1 article. Therefore, the bibliographic search of the electronic libraries consulted (PubMed and EMBASE) produced a total of 25 scientific articles (Fig. 1).

Of the 25 studies included in the analysis, 4 were clinical studies with patients, 2 were carried out in experimental animal models, and the remaining 19 were conducted in simulated environments. The greatest number of articles on the subject analyzed were published in 2015. The first study

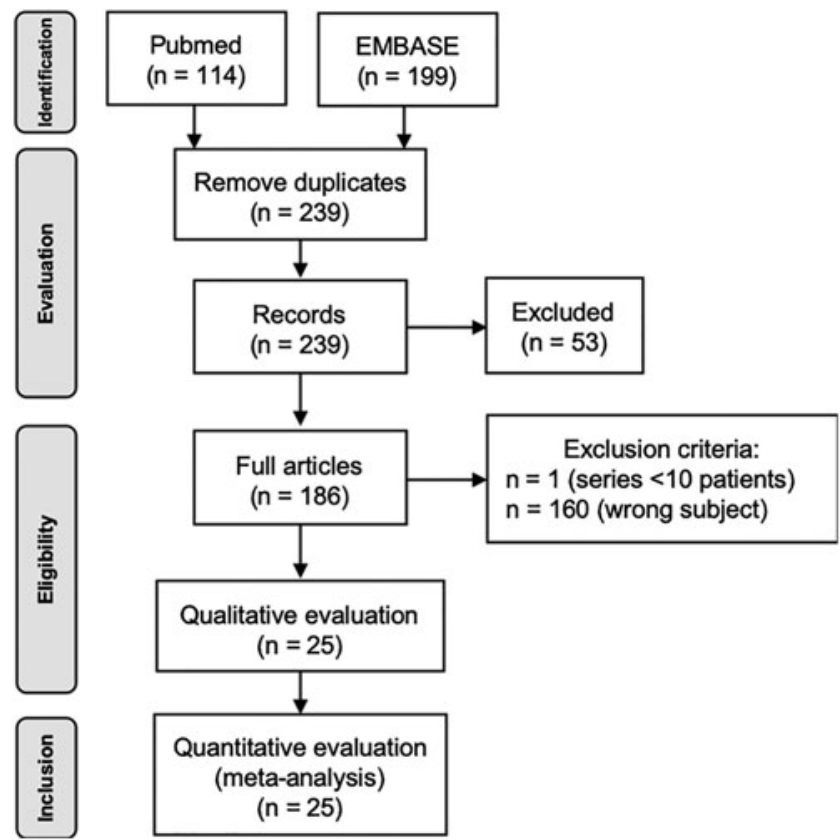

FIG. 1. Screening flowchart. 
in the scientific literature that compared the use of 2D and 3D vision systems in laparoscopic urology was from $1996 .^{12}$

The most commonly used 3D imaging systems were the Karl Storz FULL HD 3D System (Karl Storz GmbH \& Co. KG, Tüttlingen, Germany) ${ }^{13-21}$ and the Viking 3D HD Laparoscopic Vision System (Viking Systems, Inc., La Jolla, CA). ${ }^{22-30}$ Additionally, the Einstein Vision 3D (SchoellyFibreoptic GMBH, Germany) was used in some studies. ${ }^{31,32}$ Others, such as McDougall et al., conducted their studies with the Endolive Stereo Endoscope (Carl Zeiss, Oberkochen, Germany) and the StereoVu Video Laparoscopen (Welch Allyn Surgical Imaging Systems, Skaneateles Falls, NY) systems. ${ }^{12}$ Sun et al. used the DeepVision 3D video system (Automated Medical Products Corp). ${ }^{33}$ In addition, other studies used the 3D vision system implemented in the da Vinci Surgical System (Intuitive Surgical, Inc., Sunnyvale, CA). ${ }^{34-36}$

\section{Task time}

With regard to the European training program for basic laparoscopic urological skills (E-BLUS), although there was a tendency to reduce the execution time with the use of the 3D vision, for both the novice and expert surgeon groups, the meta-analysis shows no statistically significant differences in the use of either imaging system (Figs. 2 and 3).

The time required to complete the peg transfer (elastic bands and pegs), ${ }^{26,30,34}$ needle guidance, ${ }^{25,28,34}$ and cutting and suturing ${ }^{26}$ tasks was shorter for all groups of surgeons (novices, intermediates, and experts) using 3D vision (Table 1). The overall time for the fundamentals of laparoscopic surgery (FLS) tasks was better for novice surgeons with $3 \mathrm{D}$ vision than with $2 \mathrm{D}$ vision. ${ }^{31}$ In addition, $3 \mathrm{D}$ vision shortened the operative time for vesicourethral anastomosis for novices and expert surgeons. ${ }^{22,35}$ It was observed that the execution time and the accuracy of linear cutting and suturing, curved cutting and suturing, dorsal vein complex suturing, and vesicourethral anastomosis were improved for novice surgeons using $3 \mathrm{D}$ vision. ${ }^{29}$

\section{Errors}

Analyzing the errors during the E-BLUS tasks, the novice surgeons reduced the number of mistakes during the eye/hand coordination, cutting and suturing tasks when using 3D vi$\operatorname{sion}^{21,26,28,32}$ (Fig. 4). On the other hand, although the expert surgeons reduced the number of errors during the needle guidance task using 3D imaging, the novice surgeons showed an increase with respect to $2 \mathrm{D}$ vision. ${ }^{14}$ In addition, the surgeons showed fewer errors in favor of the $3 \mathrm{D}$ vision systems during the performance of peg transfer, eye/hand coordination, and cutting and suturing tasks. ${ }^{26,32}$

\section{Surgery time}

The results of the meta-analysis show that the use of 3D imaging systems led to a significant reduction in surgery time in pyeloplasty and radical nephrectomy (Fig. 5). In addition, this reduction was also reported in nephron-sparing nephrectomy and ureterolithotomy ${ }^{13}$ procedures (Table 2 ). The time required to complete the critical steps during adrenalectomy, nephron-sparing nephrectomy, radical and simple nephrectomy, pyeloplasty, and ureterolithotomy was shortened using
3D vision. ${ }^{13}$ Furthermore, a reduction in dissection time was reported for simple nephrectomy ${ }^{23}$ and pyeloplasty ${ }^{19,23}$ when using 3D vision systems. The efficiency score for pyeloplasty and partial nephrectomy was better using 3D imaging compared with conventional 2D technology. ${ }^{19}$ Similarly, novice surgical assistants performed surgical tasks more efficiently during radical prostatectomy using $3 \mathrm{D}$ vision. ${ }^{27}$ There was a reported decrease in blood loss during adrenalectomy, nephron-sparing nephrectomy, radical nephrectomy, simple nephrectomy, and pyeloplasty using $3 \mathrm{D}$ vision. ${ }^{13,23}$

\section{Ergonomics}

The studies generally reported no differences in side effects (headache, nausea, eye strain) when comparing both types of visualization systems ${ }^{22,26}$ (Table 3 ). However, the study from Gómez-Gómez et al. described significantly higher incidences of headache and visual distress using the 3D imaging system. ${ }^{17}$ In contrast, the study from Özsoy et al. reported better visual comfort when using 3D vision. ${ }^{18} \mathrm{No}-$ vice surgeons reported a significant reduction in overall workload when using $3 \mathrm{D}$ vision compared with $2 \mathrm{D}$ vision during laparoscopic training ${ }^{17,18}$ (Table 3). However, their satisfaction with the surgical performance was more favorable with $2 \mathrm{D}$ vision. ${ }^{17}$ Regarding the stress experienced by surgeons during the surgical procedures, the use of 3D imaging systems in laparoscopic urological procedures resulted in lower stress than using traditional laparoscopy. ${ }^{13,23}$

\section{Discussion}

The lack of depth perception is one of the main limitations of traditional 2D imaging systems. ${ }^{14}$ Three-dimensional vision can effectively improve spatial orientation and depth perception during laparoscopic procedures and facilitate the performance of certain complex surgical maneuvers. ${ }^{18,32}$ Following early research, ${ }^{37}$ which determined that $3 \mathrm{D}$ vision could enhance laparoscopic skills in a laboratory, many studies have been conducted to determine the contribution of $3 \mathrm{D}$ vision to laparoscopic training and surgery. ${ }^{38-40}$ However, a Cochrane review in 2011 showed no significant differences during a standard laparoscopic procedure, such as laparoscopic cholecystectomy, in the use of 3D or standard $2 \mathrm{D}$ vision. ${ }^{39}$ On the other hand, more recent studies suggest that 3D systems bring improvements to laparoscopic practice. $^{13,14,22}$ Therefore, this topic, which has been discussed since its origins, should be the subject of further research.

Of the 25 studies included in this review, most (19 in total) were carried out in a box trainer setting, including one study on single-port laparoscopic surgery ${ }^{14}$ and another combined with the ETHOS ${ }^{\mathrm{TM}}$ operating ergonomic chair (ETHOS Surgical, Portland, OR, USA). ${ }^{16}$ There were two studies on an experimental model ${ }^{12,19}$ and only four clinical studies. $^{13,15,23,27}$ Considering the above, it is clear that more clinical studies are needed in urological surgery to reinforce conclusions regarding the influence of $3 \mathrm{D}$ vision in this surgical discipline. Apart from the present analysis, we found previous studies that examined the use of 3D imaging in urological procedures but did not take into account training programs or ergonomic aspects as well as a European consensus regarding its use in different laparoscopic surgical disciplines. ${ }^{41,42}$ To the best of our knowledge, this is the first systematic review and meta-analysis carried out with respect 
Cicione et al. $2013 \quad 23182.0015 .7523209 .0019 .50$ Aykan et al. $2019 \quad 18 \quad 177.00 \quad 7.7518247 .00 \quad 1.50$ Gómez-Gómez et al. $201546 \quad 213.20 \quad 86.28 \quad 46 \quad 262.67 \quad 76.27$ Vilaça et al. $2018 \quad 14191.2093 .73 \quad 14 \quad 196.6064 .83$ Aykan et al. 2017

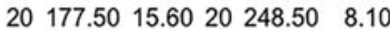

Overall effect Prediction interval Heterogeneity: $I^{2}=96 \%, \tau^{2}=24.1336, p<0.01$

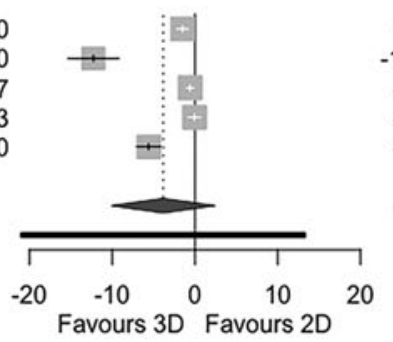

$-1.50 \quad[-2.16 ;-0.84] \quad 20.4 \%$

$-12.26[-15.33 ;-9.19] \quad 18.6 \%$

$-0.60[-1.02 ;-0.18] \quad 20.5 \%$

$-0.07 \quad[-0.81 ; 0.68] \quad 20.4 \%$

$-5.60 \quad[-7.03 ;-4.17] \quad 20.1 \%$

$-3.85[-10.06 ; 2.36] 100.0 \%$ $[-21.03 ; 13.33]$ b

Author

Cicione et al. 2013

Aykan et al. 2019

Gómez-Gómez et

Vilaça et al. 2018

Aykan et al. 2017

Overall effect

Prediction interval

Heterogeneity: $I^{2}=96 \%, \tau^{2}=39.4692, p<0.01$

Author

Cicione et al. 2013

Aykan et al. 2019

Gómez-Gómez et

Aykan et al. 2017

N Mean 3 D

2D

23223.0014 .2523228 .00117 .00

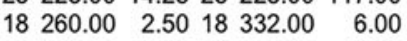
$\begin{array}{lllllll}46 & 140.67 & 49.82 & 46 & 156.37 & 42.28\end{array}$ $\begin{array}{llllll}14 & 240.80 & 85.31 & 14 & 266.60 & 57.87\end{array}$ $\begin{array}{llllll}20 & 260.50 & 8.20 & 20 & 330.00 & 19.20\end{array}$

\section{Overall effect}

Prediction interval

Heterogeneity: $I^{2}=96 \%, \tau^{2}=16.5028, p<0.01$
$N$ Mean $\quad \begin{aligned} & \text { SD } \\ & \mathrm{SD}\end{aligned}$

$23301.0042 .5023 \quad 329.00 \quad 53.25$ $\begin{array}{llllll}18 & 200.00 & 5.75 & 18 & 250.00 & 4.00\end{array}$

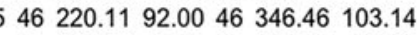
$20432.2047 .5020 \quad 588.00 \quad 30.10$

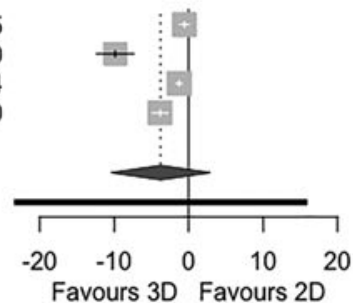

g $95 \% \mathrm{Cl}$ weight

$-0.06 \quad[-0.64 ; 0.52] 20.4 \%$

$-15.32[-19.12 ;-11.51] \quad 18.6 \%$

$-0.34[-0.75 ; 0.07] 20.4 \%$

$-0.34 \quad[-1.09 ; 0.40] \quad 20.4 \%$

$-4.61 \quad[-5.85 ;-3.38] 20.2 \%$

$-3.94[-11.86 ; 3.98] 100.0 \%$

$[-25.90 ; 18.02]$

$95 \% \mathrm{Cl}$ weight

$-0.57 \quad[-1.16 ; 0.02] 25.6 \%$

$-9.87 \quad[-12.37 ;-7.37] \quad 23.4 \%$

$-1.28 \quad[-1.73 ;-0.83] \quad 25.7 \%$

$-3.84[-4.92 ;-2.76] \quad 25.3 \%$

$-3.76[-10.37 ; 2.85] 100.0 \%$ $[-23.39 ; 15.87]$

d

Author

Cicione et al. 2013

Aykan et al. 2019

Gómez-Gómez

Vilaça et al. 2018

Overall effect

Prediction interval

Heterogeneity: $I^{2}=96 \%, \tau^{2}=24.8528, p<0.01$ $\begin{array}{lllll} & & \text { 3D } & & \text { 2D } \\ N & \text { Mean } & \text { SD } N \text { Mean } & \text { SD }\end{array}$

$\begin{array}{llllll}23 & 226.00 & 33.25 & 23 & 310.00 & 41.75\end{array}$ $\begin{array}{llllll}18 & 427.00 & 20.50 & 18 & 595.00 & 5.00\end{array}$ $\begin{array}{llllll}46 & 175.35 & 62.65 & 46 & 198.04 & 45.57\end{array}$

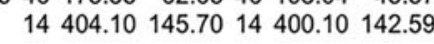

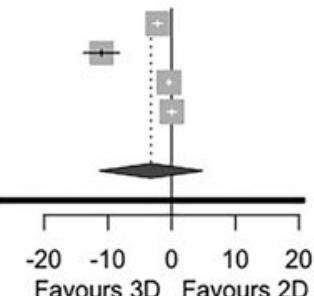

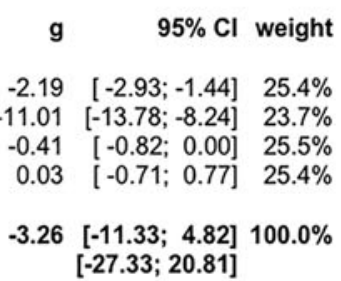

$[-27.33 ; 20.81]$ e

\begin{tabular}{|c|c|c|c|c|c|c|}
\hline Author & $\mathbf{N}$ & Mean & $\begin{array}{l}3 D \\
\text { SD }\end{array}$ & $\mathbf{N}$ & Mean & $\begin{array}{l}2 D \\
S D\end{array}$ \\
\hline Cicione et al. 2013 & 23 & 403.00 & 39.50 & 23 & 360.00 & 71. \\
\hline Aykan et al. 2019 & 18 & 842.00 & 26.75 & 18 & 1080.00 & 53. \\
\hline Gómez-Gómez et al. 2015 & 46 & 256.76 & 91.02 & 46 & 345.63 & 114 \\
\hline Vilaça et al. 2018 & 14 & 598.40 & 6.25 & 14 & 600.00 & 0. \\
\hline Aykan et al. 2017 & 20 & 200.20 & 16.70 & 20 & 249.00 & \\
\hline $\begin{array}{l}\text { Overall effect } \\
\text { Prediction interval } \\
\text { Heterogeneity: } I^{2}=97 \%, \tau^{2}\end{array}$ & • & $8, n<0$ & 0.01 & - & & \\
\hline
\end{tabular}

2D

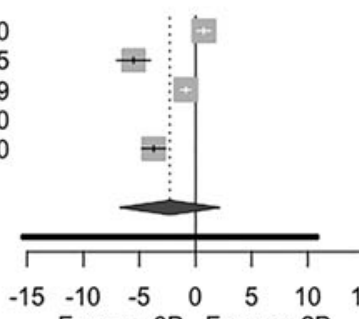

$\begin{array}{llllll}-15 & -10 & -5 & 0 & 5 & 10\end{array}$

\begin{tabular}{rrr} 
g & $95 \%$ Cl & weight \\
& & \\
0.73 & {$[0.13 ; 1.33]$} & $25.5 \%$ \\
-5.52 & {$[-7.02 ;-4.02]$} & $24.0 \%$ \\
-0.85 & {$[-1.28 ;-0.42]$} & $25.7 \%$ \\
& & $0.0 \%$ \\
-3.73 & {$[-4.79 ;-2.67]$} & $24.9 \%$ \\
-2.28 & {$[-6.74 ; 2.17]$} & $100.0 \%$ \\
& {$[-15.52 ; 10.96]$} \\
\hline
\end{tabular}

FIG. 2. Forest plots. Execution time for the E-BLUS tasks performed by novice surgeons: (a) peg transfer, (b) cut a circle, (c) knot, (d) clip and cut, and (e) needle guidance. E-BLUS, European training program in basic laparoscopic urological skills. 


Author $\quad \mathrm{N}$ Mean $\quad$ SD $\mathrm{N}$ Mean $\quad$ SD

2D

Cicione et al. $2013 \quad 10101.0011 .3210 \quad 102.00 \quad 13.00$ Aykan et al. $2019 \quad 15 \quad 85.00 \quad 10.0015 \quad 125.00 \quad 6.25$

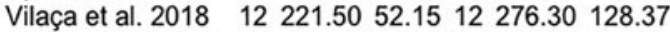

Aykan et al. $2017 \quad 20 \quad 93.7021 .50 \quad 20 \quad 128.20 \quad 25.30$

Overall effect

Prediction interval

Heterogeneity: $I^{2}=90 \%, \tau^{2}=3.7664, p<0.01$

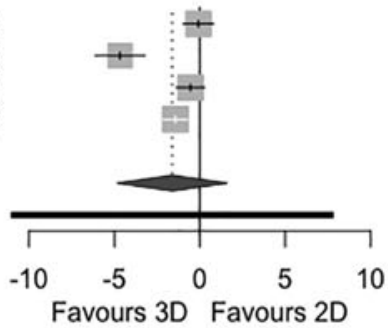

g $\quad 95 \% \mathrm{Cl}$ weight

$-0.08 \quad[-0.96 ; 0.80] \quad 25.4 \%$

$-4.67[-6.12 ;-3.21] \quad 23.3 \%$

$-0.54[-1.36 ; 0.28] \quad 25.5 \%$

$-1.44[-2.14 ;-0.74] 25.8 \%$

$-1.62[-4.84 ; 1.60] 100.0 \%$ $[-11.03 ; 7.80]$ b

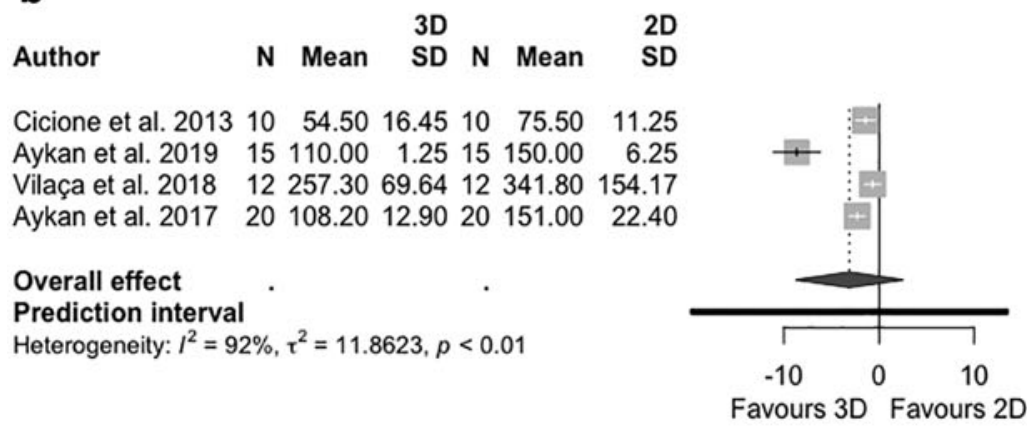

C

Author $\quad \mathrm{N}$ Mean $\quad$ SD $\mathrm{N}$ Mean $\quad$ SD

Cicione et al. $201310104.00 \quad 11.75 \quad 10 \quad 85.50 \quad 5.30$

Aykan et al. $2019 \quad 15 \quad 50.00 \quad 2.50 \quad 15 \quad 85.00 \quad 6.25$

Aykan et al. $2017 \quad 20161.7010 .7020 \quad 192.20 \quad 24.40$

Overall effect

Prediction interval

Heterogeneity: $I^{2}=97 \%, \tau^{2}=19.9505, p<0.01$

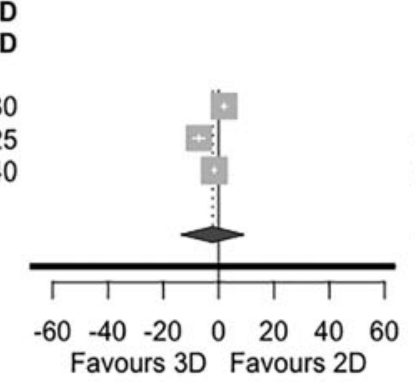

d

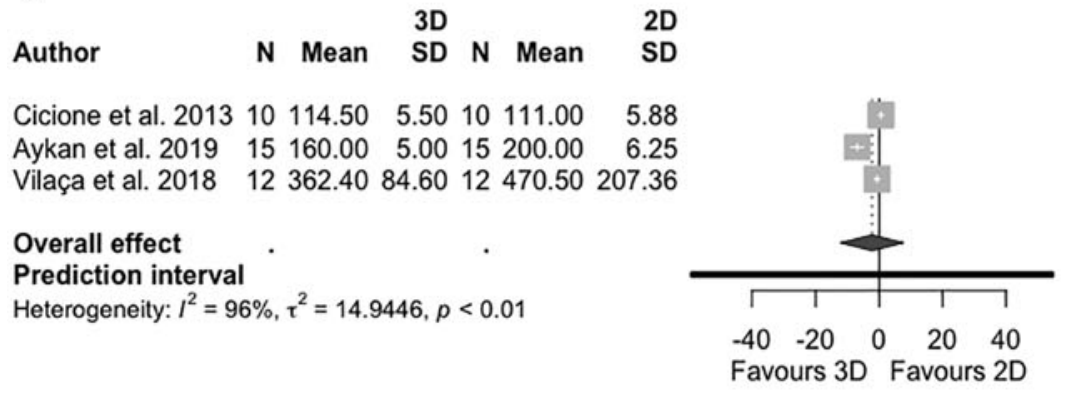

g $\quad 95 \% \mathrm{Cl}$ weight

$-1.43 \quad[-2.43 ;-0.42] \quad 25.5 \%$

$-8.64[-11.09 ;-6.18] \quad 23.1 \%$

$-0.68 \quad[-1.51 ; 0.15] \quad 25.7 \%$

$-2.30[-3.11 ;-1.48] \quad 25.7 \%$

$-3.12[-8.77 ; 2.53] 100.0 \%$ $[-19.79 ; 13.55]$

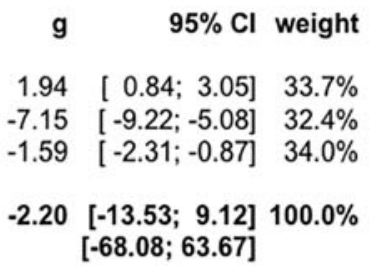

e

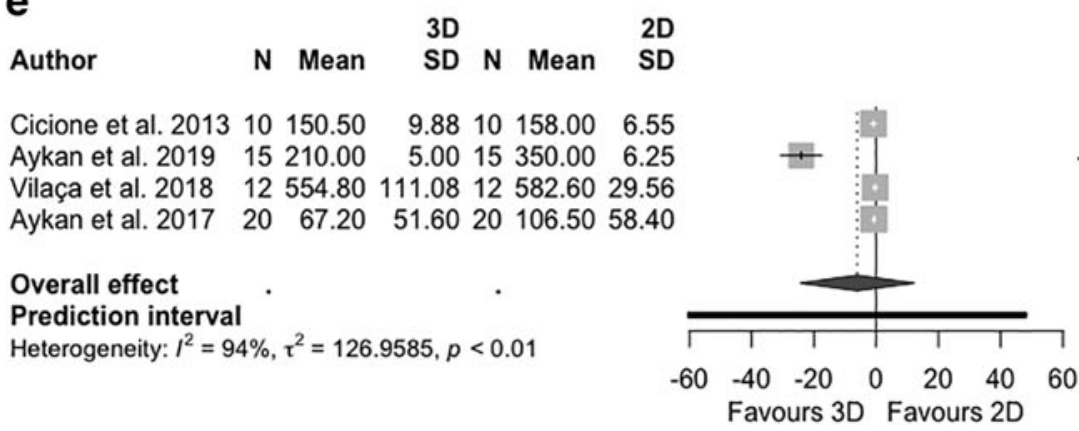

g $95 \% \mathrm{Cl}$ weight

$-0.86 \quad[-1.78 ; 0.07] \quad 25.5 \%$ $-24.07[-30.64 ;-17.49] 23.5 \%$

$-0.33[-1.14 ; 0.48] 25.5 \%$

$-0.70[-1.34 ;-0.06] 25.5 \%$

$-6.13[-24.38 ; 12.13] 100.0 \%$ $[-60.53 ; 48.27]$

FIG. 3. Forest plots. Execution time for the E-BLUS tasks performed by experienced surgeons: (a) peg transfer, (b) cut a circle, (c) knot, (d) clip and cut, and (e) needle guidance. 


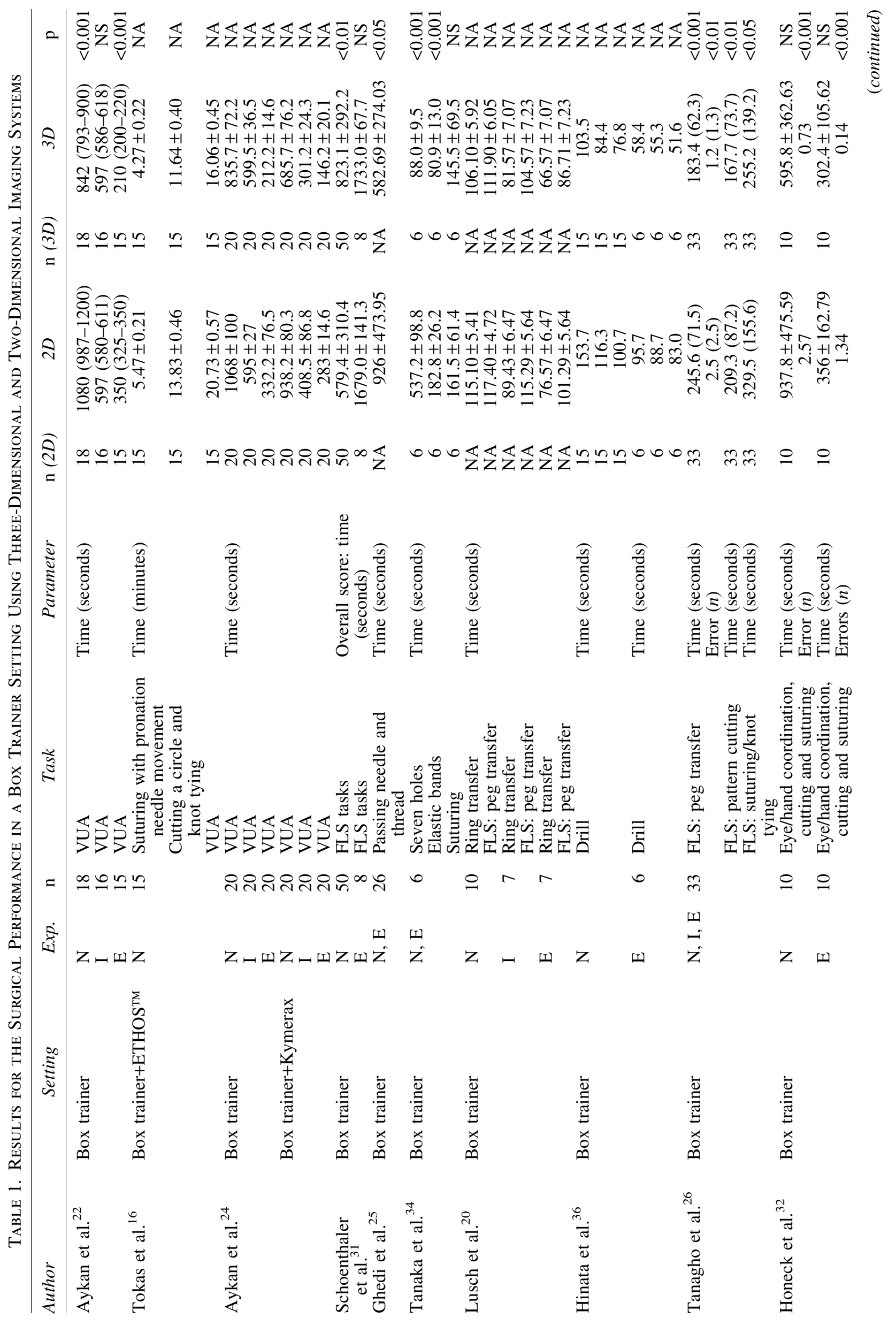




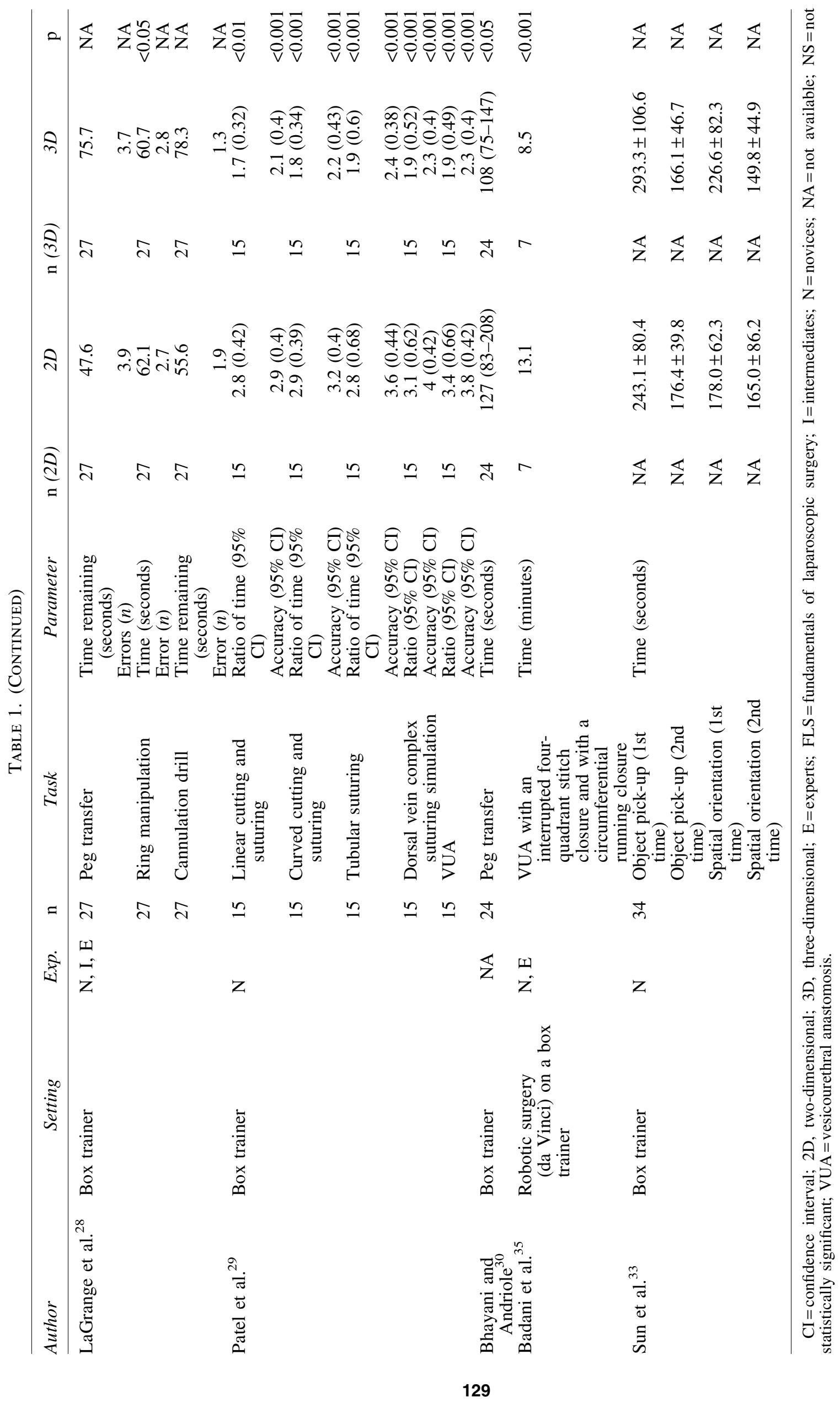


a

Author

$\begin{array}{lrlllll}\text { Cicione et al. } 2013 & 23 & 0.00 & 0.25 & 23 & 1.00 & 0.50 \\ \text { Gómez-Gómez et al. } 2015 & 46 & 0.30 & 0.59 & 46 & 0.69 & 0.81\end{array}$

Vilaça et al. 2018

$\begin{array}{llllll}14 & 0.94 & 1.06 & 14 & 0.62 & 1.09\end{array}$

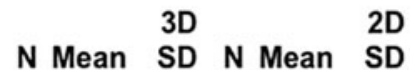

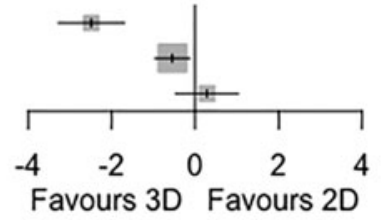

b

Author

Cicione et al. $2013 \quad 23 \quad 0.00 \quad 0.0023 \quad 1.00 \quad 0.50$

Gómez-Gómez et al. $\begin{array}{lllllll}2015 & 46 & 0.02 & 0.14 & 46 & 0.07 & 0.25\end{array}$

Vilaça et al. 2018

$\begin{array}{llllll}14 & 0.75 & 1.34 & 14 & 1.44 & 1.26\end{array}$

C

Author

N Mean SD $\mathrm{N}$ Mean SD

Cicione et al. 2013

$\begin{array}{llllll}23 & 1.00 & 0.25 & 23 & 1.00 & 0.50\end{array}$

Gómez-Gómez et al. $201546 \quad 0.24 \quad 0.56 \quad 46 \quad 0.87 \quad 1.06$

d

Author

3D

Cicione et al. 2013

Gómez-Gómez et al. 2015

Vilaça et al. 2018

$\begin{array}{llllll}23 & 1.00 & 0.25 & 23 & 2.00 & 0.75\end{array}$

$\begin{array}{llllll}46 & 0.28 & 0.62 & 46 & 0.24 & 0.48\end{array}$

$\begin{array}{llllll}14 & 0.06 & 0.25 & 14 & 0.38 & 0.72\end{array}$

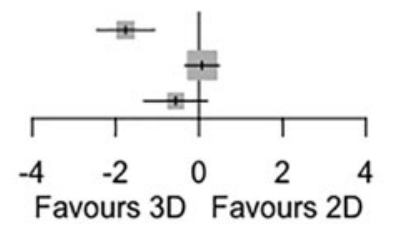

e

Author

3D

$\mathrm{N}$ Mean SD $\mathrm{N}$ Mean SD

Cicione et al. 2013

$\begin{array}{llllll}23 & 0.00 & 0.00 & 23 & 0.00 & 0.00\end{array}$

Gómez-Gómez et al. $201546 \quad 0.00 \quad 0.0046 \quad 0.00 \quad 0.00$

Vilaça et al. 2018

\section{D}
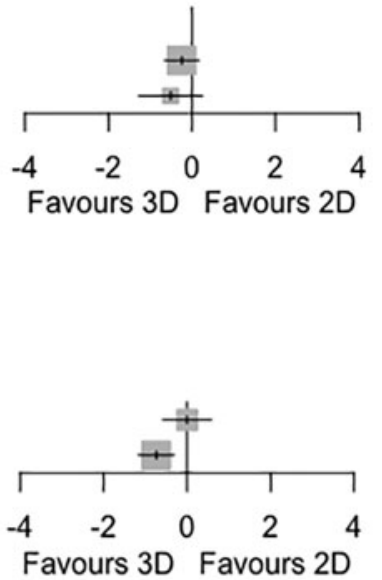

SMD $\quad 95 \%-\mathrm{Cl}$

$-1.76[-2.45 ;-1.07]$

$0.07[-0.34 ; 0.48]$

$-0.56[-1.32 ; 0.19]$

Favours 3D Favours 2D

(2)
SMD

$95 \%-\mathrm{Cl}$

$-0.24[-0.65 ; 0.17]$

$-0.51[-1.27 ; 0.24]$

SMD $\quad 95 \%-\mathrm{Cl}$

$0.00[-0.58 ; 0.58]$

$-0.74[-1.16 ;-0.31]$

$-2.49[-3.27 ;-1.70]$

$0.28[-0.46 ; 1.03]$

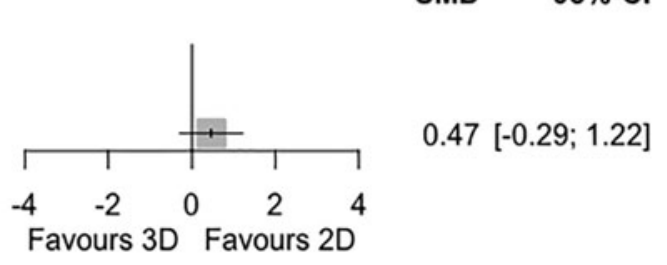

FIG. 4. Forest plots. Errors for the E-BLUS tasks performed by novice surgeons: (a) peg transfer, (b) cut a circle, (c) knot, (d) clip and cut, and (e) needle guidance. Summary (meta-analytic) values are omitted due to the dissimilarities of the studies.

to surgical performance and the surgeon's ergonomics in 3D laparoscopic urological surgery, including both training tasks and surgical procedures.

The European Association of Endoscopic Surgery (EAES) presented in 2018 a consensus to establish a set of evidencebased statements and recommendations for the use of $3 \mathrm{D}$ vision in laparoscopy. ${ }^{41}$ They included randomized con- trolled trials and prospective studies, addressing different subjects such as general topics, organ-specific data, and ongoing trials. For the organ-specific studies, the impact of 3D vision on operative time and complications were mainly analyzed. Dire et al. also examined the effect of the use of this vision technology on operating time and estimated blood loss in urological procedures. ${ }^{42}$ However, the procedures were 

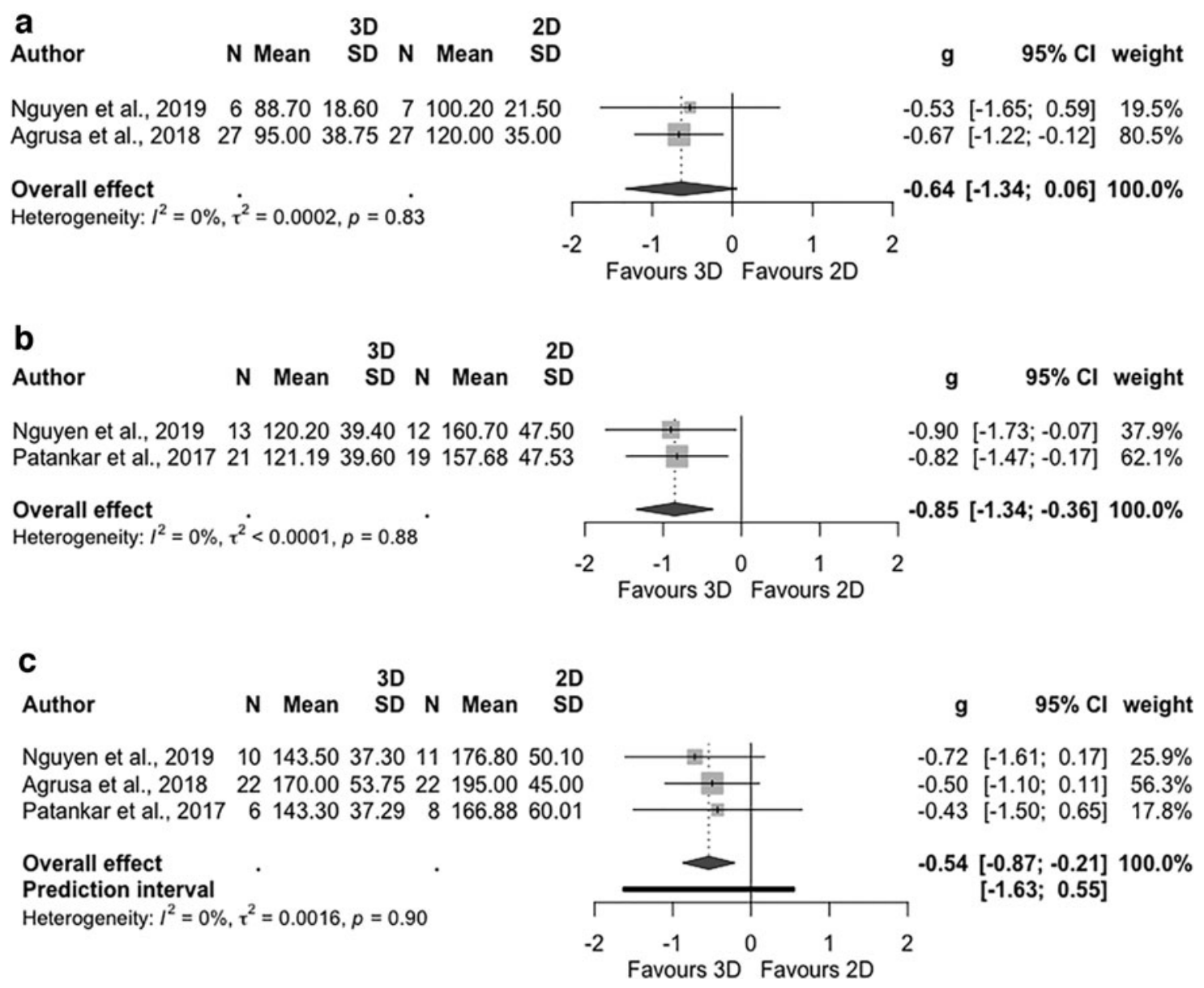

FIG. 5. Forest plots. Surgery time for (a) adrenalectomy, (b) pyeloplasty, and (c) radical nephrectomy using 3D and 2D imaging systems. 2D, two-dimensional; 3D, three-dimensional.

limited to partial nephrectomy, pyeloplasty, and radical prostatectomy. Laparoscopic training tasks, execution errors, and ergonomic parameters were not taken into account.

It seems that laparoscopic 3D imaging may be beneficial during the initial learning process, especially for less advanced surgeons using physical simulators. The results showed that, in general, the use of $3 \mathrm{D}$ vision technology led to a reduction in the execution time for laparoscopic training tasks. ${ }^{19}$ The time required to complete eye/hand coordination tasks such as peg transfer, elastic bands, or needle guidance was shorter using $3 \mathrm{D}$ vision compared with conventional $2 \mathrm{D} .^{25,26,28,30,34}$ In addition, the number of achieved goals and transferred objects during the same period of time were also higher during needle guidance and peg transfer tasks, respectively. ${ }^{18}$

The use of 3D vision technology clearly provides better depth perception and spatial location and therefore assists in the execution of eye/hand coordination tasks. Similarly, it was reported that surgeons required less time, improving cutting accuracy and average cutting time using 3D imaging systems. ${ }^{18,22,26,29}$ Regarding the suturing tasks, such as knot tying, simple suturing or complex suturing, 3D vision improved the surgical performance regardless of the experience of the surgeon. These improvements were mainly in the number of knots, execution time, and accuracy. ${ }^{18,22,26,29}$

In general, surgeons showed an improvement in the execution time established in the European training in basic laparoscopic urological skills (E-BLUS) program when using 3D vision. ${ }^{21}$ These results were remarkable during eye/hand coordination tasks such as peg transfer or needle guidance, ${ }^{18,22,25,26,28-30,34}$ which were mainly designed to assess ambidexterity, among other surgical skills. ${ }^{43}$ Novice and expert surgeons also required less time to complete vesicourethral anastomosis compared with conventional 2D vision, ${ }^{22,35}$ showing a higher procedural accuracy among the novice group. ${ }^{29}$

In the case of the FLS program, there was a low number of experienced participants. Only one study examined the overall execution time, ${ }^{31}$ which was significantly improved in the group of novice surgeons using 3D vision. That expert surgeons had similar results using $2 \mathrm{D}$ and $3 \mathrm{D}$ vision in the development of basic laparoscopic tasks could be due to having been trained mainly with traditional 2D imaging systems, which has given them certain skills to overcome several of its limitations.

There was an overall reduction in the number of errors made in eye/hand coordination, and cutting and suturing tasks using $3 \mathrm{D}$ vision technology. ${ }^{18,21,26,28,32}$ This may be due to improvements in depth and spatial orientation over traditional 2D displays. Another study also stated that the use of 3D imaging systems improves laparoscopic box trainer task completion time and error rate. ${ }^{41}$

Studies involving the E-BLUS program showed a high disparity in the type of tasks used, thereby increasing the 


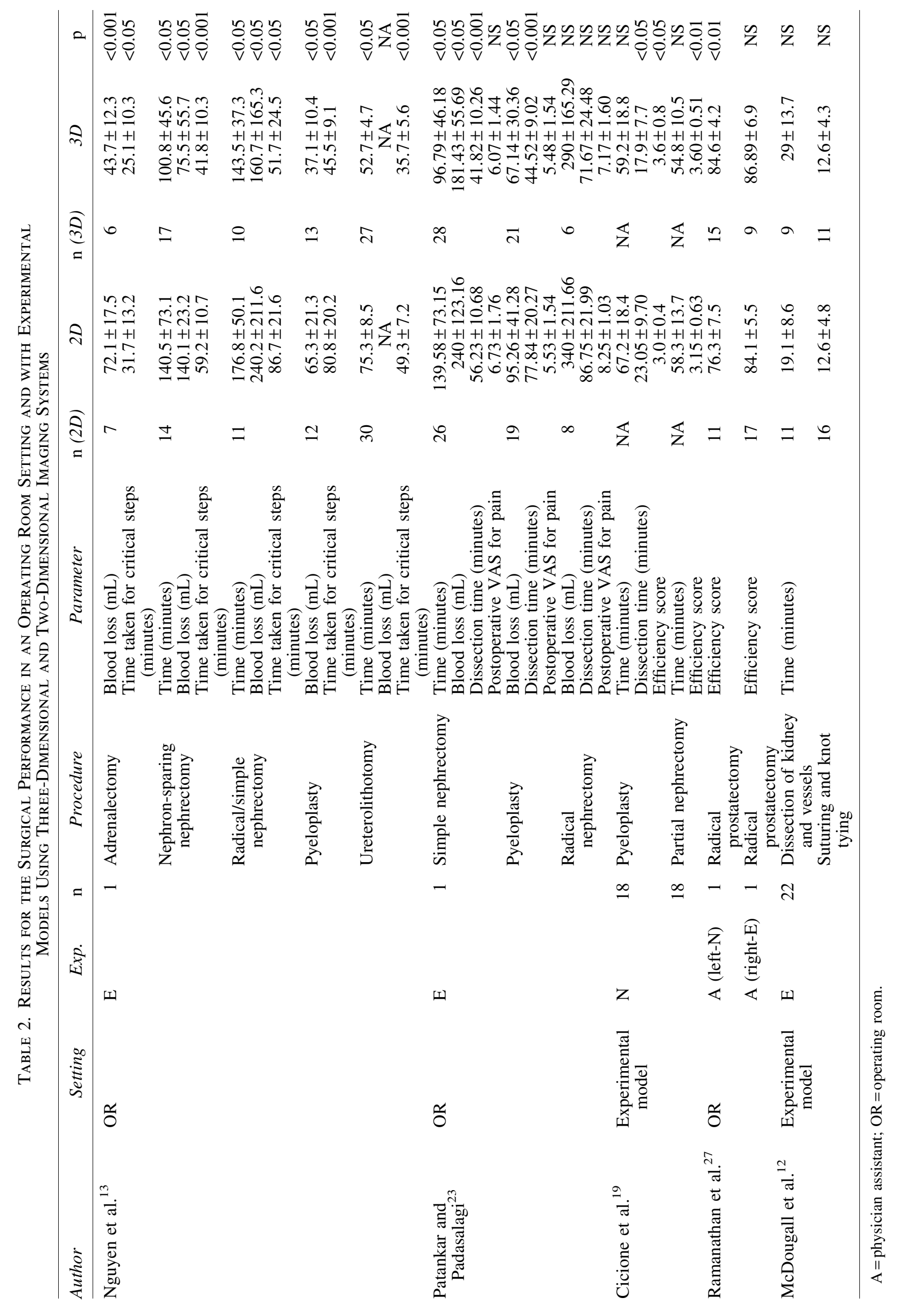


Table 3. Results for the Surgeon's Ergonomic Factors Using Three-Dimensional and Two-Dimensional Imaging Systems

\begin{tabular}{|c|c|c|}
\hline Author & Evaluation & Results \\
\hline Aykan et al. ${ }^{22}$ & $\begin{array}{l}\text { Personalized questionnaire (2D vs 3D; } \\
\text { score: } 1-5 \text { ) } \\
\text { Muscular pain (2D vs 3D; score: } 1-5) \\
\text { Handgrip strength (2D vs 3D) }\end{array}$ & $\begin{array}{l}\text { No differences in side effects (dizziness, } \\
\text { blur, feeling shaky, and nausea) } \\
\text { Less with 3D system*** } \\
1.35(0.9-2.1) \text { vs } 0.75(0.5-1.2)^{*}\end{array}$ \\
\hline Nguyen et al. ${ }^{13}$ & Personalized questionnaire (3D) & $\begin{array}{l}\text { No side effects (headache, nausea, and eye } \\
\text { strain) }\end{array}$ \\
\hline Tokas et al. ${ }^{16}$ & $\begin{array}{l}\text { Personalized questionnaire (2D vs } \\
\text { ETHOS }{ }^{\mathrm{TM}}+3 \mathrm{D} \text {; heavy discomfort) }\end{array}$ & $\begin{array}{l}\text { Suturing with pronation needle movement: } \\
80 \% \text { vs } 6.7 \% * * * \\
\text { Suturing on a tubular structure: } 86.7 \% \text { vs } \\
13.3 \% * * * \\
\text { VUA: } 86.7 \% \text { vs } 20 \% * * *\end{array}$ \\
\hline Patankar and Padasalagi ${ }^{23}$ & STAI-6 (2D vs 3D) & $\begin{array}{l}\text { Simple nephrectomy: } 16.54 \pm 2.44 \text { vs } \\
13.39 \pm 2.75^{* * *} \\
\text { Pyeloplasty: } 16.16 \pm 3.53 \text { vs } 12.67 \pm 2.27 * * * \\
\text { Radical nephrectomy: } 19.88 \pm 2.75 \mathrm{vs} \\
\quad 15.33 \pm 3.08^{*}\end{array}$ \\
\hline Gómez-Gómez et al. ${ }^{17}$ & $\begin{array}{l}\text { NASA-TLX (2D vs 3D) } \\
\text { Personalized questionnaire (2D vs 3D; } \\
\quad \text { score: } 0-20)\end{array}$ & $\begin{array}{l}62.10 \pm 12.59 \text { vs } 53.89 \pm 13.08 * * * \\
\text { Visual discomfort: } 3.78 \pm 4.32 \text { vs } \\
\quad 6.86 \pm 5.82^{*} \\
\text { Headache: } 1.22 \pm 1.91 \text { vs } 2.91 \pm 4.76^{*}\end{array}$ \\
\hline Özsoy et al. ${ }^{18}$ & $\begin{array}{l}\text { NASA-TLX (2D vs 3D; median [IQR]) } \\
\text { Personalized questionnaire (2D vs 3D; } \\
\quad \text { median [IQR]) }\end{array}$ & $\begin{array}{l}72.30[25.10] \text { vs } 60.00[19.60]^{* * *} \\
\text { Depth perception: } 2.00[1.00] \text { vs } 4.00 \\
\quad[0.00]^{* * *} \\
\text { Visual comfort: } 3.00[1.00] \text { vs } 4.00 \\
\quad[2.00]^{* *}\end{array}$ \\
\hline Cicione et al. ${ }^{19}$ & Personalized questionnaire (3D) & $\begin{array}{l}18.1 \% \text { of side effects (headache, nausea, and } \\
\text { visual disturbance) }\end{array}$ \\
\hline Tanaka et al. ${ }^{34}$ & $\begin{array}{l}\text { Personalized questionnaire (3D; score: } \\
\quad 0-100)\end{array}$ & $\begin{array}{l}\text { Vision: } 87.7 \pm 7.1 \\
\text { Weight of the device: } 19.2 \pm 7.9 \\
\text { Visual disturbance: } 7.5 \pm 6.1 \\
\text { Eyestrain: } 24.2 \pm 7.9\end{array}$ \\
\hline Tanagho et al. ${ }^{26}$ & Personalized questionnaire (2D vs 3D) & $\begin{array}{l}\text { No differences in side effects (eye strain, } \\
\text { headache, dizziness, disorientation, } \\
\text { physical discomfort, and poor } \\
\text { visualization) }\end{array}$ \\
\hline Ramanathan et al. ${ }^{27}$ & $\begin{array}{l}\text { Personalized questionnaire (3D; score: } \\
\quad 0-100)\end{array}$ & $\begin{array}{l}\text { Vision: } 90 \\
\text { Weight of the device: } 65 \\
\text { Restrictions of the field of view: } 25\end{array}$ \\
\hline McDougall et al. ${ }^{12}$ & Personalized questionnaire (3D) & $\begin{array}{l}\text { Cumbersome glasses: } 53 \% \\
\text { Heavy glasses: } 36 \% \\
\text { Adverse effects: } 12 \%\end{array}$ \\
\hline
\end{tabular}

$* p<0.05, * * p<0.01, * * * p<0.001$

$\mathrm{IQR}=$ interquartile range; NASA-TLX=NASA task load index; STAI-6=State-Trait Anxiety Inventory for Adults.

variability of the results obtained and hampering the statistical analysis (meta-analysis) (Fig. 4). Overall, a reduction in the number of errors was shown when using $3 \mathrm{D}$ vision. ${ }^{17,22}$ Specifically, novice surgeons made fewer errors using 3D imaging systems, mainly in tasks that were more demanding and required greater depth perception (suture and needle guidance). ${ }^{17}$ Experienced surgeons showed no differences in errors with either imaging system. ${ }^{14,21}$ This demonstrates that the use of 3D vision may not be a critical factor in performing standardized urological training tasks for surgeons with previous laparoscopic backgrounds.

All analyzed studies conducted in a clinical setting or with an experimental animal model were performed by experienced laparoscopic surgeons. One study in a porcine model also included 18 surgeons without any previous laparoscopic experi- ence who had attended a training course in urological surgery. ${ }^{19}$ The use of 3D vision was shown to allow surgeons to reduce the surgery time of adrenalectomy, nephron-sparing nephrectomy, simple and radical nephrectomy, pyeloplasty, and ureterolithotomy. ${ }^{13,23}$ In particular, the time spent performing critical steps, such as dissection of the renal pedicle, needle position and driving, suture placement and tissue edge holding, was reduced compared with the use of conventional laparoscopic vision. ${ }^{13,19,23}$ It seems that the use of 3D imaging systems leads to improvements in surgery time mainly in surgical procedures involving intracorporeal ligatures and sutures. This imaging technology enables surgeons to perform complicated and crucial surgical maneuvers more quickly and safely. ${ }^{15,19,44}$

The present review included one study with radical prostatectomies, ${ }^{27}$ but no information on surgery time was 
reported. Nevertheless, other studies have shown a significant reduction in operating time in this procedure using $3 \mathrm{D}$ imaging systems. ${ }^{41,42}$ Unlike the present study, Dire et al. reported no significant difference in surgery time for pyeloplasty using $3 \mathrm{D}$ and $2 \mathrm{D}$ vision. $^{42}$ One of the three studies included was a retrospective study in children and another was a study in Chinese, which were not included in the current systematic review.

Some studies reported a reduction in blood loss for adrenalectomy, simple nephrectomy, pyeloplasty, and nephronsparing nephrectomy procedures with 3D imaging systems. ${ }^{13,23}$ According to a number of studies, this type of imaging technology makes it possible to better identify vascular structures, which allows for enhanced dissections and therefore less blood loss and improved bleeding control. ${ }^{13,23}$ As previously mentioned, 3D vision improves eye/hand coordination and depth perception, which implies an amelioration in the identification of the different anatomical structures and thus in the precision of the execution of the different surgical steps. ${ }^{23}$

Studies have shown that $3 \mathrm{D}$ vision improves the efficiency of novice surgeons, including surgical assistants, during radical prostatectomy, pyeloplasty, and partial nephrectomy. ${ }^{19,27}$ This group of surgeons appeared to experience improvements in accuracy and efficiency during laparoscopic urologic surgery using these types of vision systems. ${ }^{45}$

Ergonomics has been mainly assessed by means of subjective assessment methods such as NASA task load index (NASA-TLX), State-Trait Anxiety Inventory for Adults (STAI-6), and personalized questionnaires. Only the study of Aykan et al. ${ }^{22}$ included an objective measurement to analyze handgrip strength after the completion of tasks using 2D and 3D imaging systems. It would have been desirable to have carried out these measures during the development of the activities instead. Performing an objective assessment with regard to the effect that novel medical equipment has on the surgical working environment is a crucial aspect for assessing its adequate adaptation to the clinical requirements and its benefits and limitations during its use.

The application of ergonomic principles in surgery is fundamental for the implantation of new medical technology, mainly due to frequent complaints from laparoscopic surgeons. However, an adequate and comprehensive objective methodology has yet to be standardized. Some groups are working on defining a standard framework and objective assessment method for ergonomic analysis in laparoscopic surgery. ${ }^{46-48}$

Taking into account the subjective evaluation regarding the use of 3D imaging systems in urological laparoscopy, improvements in surgical performance and greater comfort for the surgeons have been reported, ${ }^{23}$ indicating the suitability of this technology for its use in advanced surgical conditions, in reduced spaces and in complex surgeries. ${ }^{15}$ Image quality and depth perception were also positively evaluated with $3 \mathrm{D}$ vision systems. ${ }^{13}$

During laparoscopic training, novice surgeons reported a significant reduction in general workload when using 3D vision compared with $2 \mathrm{D}$ vision. ${ }^{17,18}$ However, their satisfaction with surgical performance was more favorable using $2 \mathrm{D}$ vision. ${ }^{17}$ This result is somewhat contradictory, as the participants obtained a remarkable reduction in execution time with the use of the $3 \mathrm{D}$ vision system. ${ }^{17}$ This surgeon's perception of surgical performance could probably improve after a period of comprehensive training with this new technology. Arezzo et al. also stated that 3D laparoscopy may result in decreased experienced cognitive workload provided that the viewing setup is optimal. ${ }^{41}$

In terms of the stress experienced by surgeons during laparoscopic urological procedures, the use of 3D systems has resulted in less stress than traditional laparoscopy. ${ }^{13,23}$ This may be because 3D imaging addresses the lack of depth perception in conventional laparoscopic surgery, which may have led to a decrease in the stress caused during surgery.

Overall, no differences in side effects (headache, nausea, eye strain) were observed using both $3 \mathrm{D}$ and $2 \mathrm{D}$ vision systems during surgery. ${ }^{13,15,22,23,26}$ However, some studies reported an increase in headache and visual discomfort with the use of 3D imaging systems. ${ }^{17,19}$ These side effects may be due to the need to wear special glasses to perceive the $3 \mathrm{D}$ effect. Some studies also pointed out that the weight of the 3D vision glasses is a problem that should be addressed, although this problem currently seems to have been solved. ${ }^{12,27}$

There are clear differences in the cost of 2D and 3D laparoscopy equipment. In general, $3 \mathrm{D}$ vision equipment is more expensive than 2D. ${ }^{49}$ Although the purchase of a 3D imaging system can be up to $66 \%$ more expensive than a conventional 2D laparoscopic system, ${ }^{50}$ eventually $3 \mathrm{D}$ imaging systems have shown a considerable benefit in terms of blood loss, operative time, and hospital stay, resulting in a reduction of the costs associated with the intervention. ${ }^{50,51}$ In addition, improved depth perception, surgical precision and hand/eye coordination with 3D laparoscopic systems allow for reduced surgery time, thus reducing cost, exposure time to anesthesia and morbidity. ${ }^{51,52}$ This means better patient safety and therefore shorter hospitalization time and lower costs related to postoperative hospital stay. ${ }^{51}$ However, further studies and progress are needed to make the $3 \mathrm{D}$ imaging systems more cost effective.

Imaging and display technology are paramount in robotassisted systems providing the primary method of feedback to the surgeon. In certain cases, the implementation of $3 \mathrm{D}$ vision in surgical robotic systems differs from the one used in a conventional laparoscopic approach. The da Vinci System is the most widespread surgical robotic platform, which uses a stereoscopic vision system with dual independent displays, one for each eye. This robotic platform has no force feedback, so visual feedback is vital for the surgeon. Ques such as shadows, motion parallax, and binocular cues are used to estimate location in 3D space of the end effectors, while tissue deformation is used to estimate gripping and prodding force being applied. ${ }^{53,54}$ Currently, there are other robotic systems such as Hugo (Medtronic, Dublin, Ireland), Senhance $^{\mathrm{TM}}$ (TransEnterix, Morrisville, NC), and Versius ${ }^{\mathrm{TM}}$ (CMR Surgical, Cambridge, UK), among others that use flat panel polarized 3D display technology for visualization of the intervention workspace. ${ }^{53,55,56}$ Several studies have reported the added value of $3 \mathrm{D}$ vision in robotic surgery compared with conventional $2 \mathrm{D}$ vision. They showed that the use of 3D visualization improves the efficiency of surgical skills and reduce performance time, ${ }^{34}$ improving surgical performance during anastomosis. ${ }^{35,57}$

The benefits of 3D vision in surgical performance during standardized urological training tasks were more evident in the group of surgeons without a background in laparoscopic surgery. The results showed that the use of 3D imaging 
systems leads to improvements in surgery time, mainly in surgical procedures involving intracorporeal ligatures and sutures. Similarly, this type of imaging technology facilitates the identification of anatomical structures, enhancing the precision of maneuvers and the control of blood loss during urological procedures. With respect to surgeons' ergonomics, $3 \mathrm{D}$ vision systems provide better depth perception and reduce stress and general workload during surgery compared with traditional vision systems with no differences in potential side effects. Studies in urological surgery with this type of technology are still scarce. Further research is needed to draw more robust evidence-based findings regarding the implications of the use of 3D vision in laparoscopic urological surgery.

This review has some limitations. The number of studies included in the systematic review is limited, especially the number of randomized controlled trials in laparoscopic urological procedures. Although 3D vision has been widely implemented in surgical robotics, only three studies in this review involved robotic surgery, two of which investigated the use of the 3D technology for the bedside assistant. In addition, there is a lack of studies that apply objective assessment methods to the surgeon's ergonomics. Most studies used subjective questionnaires regarding experience in the use of this vision technology.

\section{Conclusions}

The benefits of 3D vision in improving surgical performance in standardized urological training tasks are more evident in the group of surgeons without a background in laparoscopic surgery. The results showed that the use of 3D imaging systems leads to improvements in surgery time, mainly in surgical procedures involving intracorporeal ligatures and sutures. With respect to surgeons' ergonomics, 3D vision provides better depth perception and reduces stress and workloads during surgery compared with traditional vision systems with no differences in potential side effects.

\section{Acknowledgment}

The authors specially thank Eva Sequeira for her technical support.

\section{Author Disclosure Statement}

No competing financial interests exist.

\section{Funding Information}

This work has been funded by the Government of Extremadura (Spain) (GR18199, TA18023) (cofunded by European Regional Development Fund). The funders had no role in study design, data collection, interpretation and analysis, decision to publish, or preparation of the article.

\section{References}

1. McGuinness LA, Prasad Rai B. Robotics in urology. Ann R Coll Surg Engl 2018;100(6_sup):38-44.

2. Kyriazis I, Özsoy M, Kallidonis P, Vasilas M, Panagopoulos V, Liatsikos E. Integrating three-dimensional vision in laparoscopy: The learning curve of an expert. J Endourol 2015;29:657-660.
3. Autorino R, Zargar H, Kaouk JH. Robotic-assisted laparoscopic surgery: Recent advances in urology. Fertil Steril 2014;102:939-949.

4. Tsui C, Klein R, Garabrant M. Minimally invasive surgery: National trends in adoption and future directions for hospital strategy. Surg Endosc 2013;27:2253-2257.

5. Tan HJ, Wolf JS, Jr., Ye Z, Hafez KS, Miller DC. Population level assessment of hospital based outcomes following laparoscopic versus open partial nephrectomy during the adoption of minimally invasive surgery. J Urol 2014;191:1231-1237.

6. Luketich JD, Pennathur A, Awais O, Levy RM, Keeley S, Shende M, et al. Outcomes after minimally invasive esophagectomy: Review of over 1000 patients. Ann Surg 2012;256:95-103.

7. Sánchez-Margallo FM, Diaz-Guemes I, Sánchez-Hurtado MA, Sánchez-Fernandez J, Usón Gargallo J, Serrano Pascual A. [Training in basic and advanced laparoscopy: Evaluation of a 30-year experience in a training program in Spain.]. Arch Esp Urol 2018;71:63-72.

8. Bach T, Muschter R, Herrmann TR, Knoll T, Scoffone CM, Laguna MP, et al. Technical solutions to improve the management of non-muscle-invasive transitional cell carcinoma: Summary of a European Association of Urology Section for Uro-Technology (ESUT) and Section for UroOncology (ESOU) expert meeting and current and future perspectives. BJU Int 2015;115:14-23.

9. Rassweiler JJ, Teber D. Advances in laparoscopic surgery in urology. Nat Rev Urol 2016;13:387-399.

10. Velayutham V, Fuks D, Nomi T, Kawaguchi Y, Gayet B. $3 \mathrm{D}$ visualization reduces operating time when compared to high-definition 2D in laparoscopic liver resection: A casematched study. Surg Endosc 2016;30:147-153.

11. Moher D, Shamseer L, Clarke M, Ghersi D, Liberati A, Petticrew M, et al. Preferred reporting items for systematic review and meta-analysis protocols (PRISMA-P) 2015 statement. Syst Rev 2015;4:1.

12. McDougall EM, Soble JJ, Wolf JS, Jr., Nakada SY, Elashry OM, Clayman RV. Comparison of three-dimensional and two-dimensional laparoscopic video systems. J Endourol 1996;10:371-374.

13. Nguyen DH, Nguyen BH, Van Nong H, Tran TH. Threedimensional laparoscopy in urology: Initial experience after 100 cases. Asian J Surg 2019:42:303-306.

14. Vilaça J, Leite M, Correia-Pinto J, Hogemann G, Costa P, Leao P. The influence of 3D in single-port laparoscopy surgery: An experimental study. Surg Laparosc Endosc Percutan Tech 2018;28:261-266.

15. Agrusa A, Di Buono G, Buscemi S, Cucinella G, Romano G, Gulotta G. 3D laparoscopic surgery: A prospective clinical trial. Oncotarget 2018;9:17325-17333.

16. Tokas T, Gozen AS, Avgeris M, Tschada A, Fiedler M, Klein $\mathrm{J}$, et al. Combining of ETHOS operating ergonomic platform, three-dimensional laparoscopic camera, and radius surgical system manipulators improves ergonomy in urologic laparoscopy: Comparison with conventional laparoscopy and da Vinci in a Pelvi trainer. Eur Urol Focus 2017;3:413-420.

17. Gómez-Gómez E, Carrasco-Valiente J, Valero-Rosa J, Campos-Hernández, Anglada-Curado FJ, Carazo-Carazo $\mathrm{JL}$, et al. Impact of 3D vision on mental workload and laparoscopic performance in inexperienced subjects. Actas Urol Esp 2015;39:229-235.

18. Özsoy M, Kallidonis P, Kyriazis I, Panagopoulos V, Vasilas M, Sakellaropoulos GC, et al. Novice surgeons: Do 
they benefit from 3D laparoscopy? Lasers Med Sci 2015; 30:1325-1333.

19. Cicione A, Autorino R, Laguna MP, De Sio M, Micali S, Turna B, et al. Three-dimensional technology facilitates surgical performance of novice laparoscopy surgeons: A quantitative assessment on a porcine kidney model. Urology 2015;85:1252-1256.

20. Lusch A, Bucur PL, Menhadji AD, Okhunov Z, Liss MA, Perez-Lanzac A, et al. Evaluation of the impact of threedimensional vision on laparoscopic performance. J Endourol 2014;28:261-266.

21. Cicione A, Autorino R, Breda A, De Sio M, Damiano R, Fusco F, et al. Three-dimensional vs standard laparoscopy: Comparative assessment using a validated program for laparoscopic urologic skills. Urology 2013;82:1444-1450.

22. Aykan S, Temiz MZ, Duymaz T, Ural IH, Colakerol A, Muslumanoglu AY, Semercioz A. Effects of the threedimensional vision system on surgical performance, muscular fatigue, and pain during urologic laparoscopic tasks: Results of objective assessments and a mini questionnaire survey. J Laparoendosc Adv Surg Tech A 2019;29:346-352.

23. Patankar SB, Padasalagi GR. Three-dimensional versus two-dimensional laparoscopy in urology: A randomized study. Indian J Urol 2017;33:226-229.

24. Aykan S, Akin Y, Pelit ES, Gulmez H, Tuken M, Colakerol $\mathrm{A}$, et al. Impact of motorized articulating laparoscopic devices with three-dimension visualizing system: A pilot study. J Endourol 2017;31:174-179.

25. Ghedi A, Donarini E, Lamera R, Sgroi G, Turati L, Ercole C. 3D vs 2D laparoscopic systems: Development of a performance quantitative validation model. Conf Proc IEEE Eng Med Biol Soc 2015;2015:6884-6887.

26. Tanagho YS, Andriole GL, Paradis AG, Madison KM, Sandhu GS, Varela JE, et al. 2D versus 3D visualization: Impact on laparoscopic proficiency using the fundamentals of laparoscopic surgery skill set. J Laparoendosc Adv Surg Tech A 2012;22:865-870.

27. Ramanathan R, Salamanca JI, Mandhani A, Leung RA, Rao SR, Berryhill R, et al. Does 3-Dimensional (3-D) visualization improve the quality of assistance during robotic radical prostatectomy? World J Urol 2009;27:95-99.

28. LaGrange CA, Clark CJ, Gerber EW, Strup SE. Evaluation of three laparoscopic modalities: Robotics versus threedimensional vision laparoscopy versus standard laparoscopy. J Endourol 2008;22:511-516.

29. Patel HR, Ribal MJ, Arya M, Nauth-Misir R, Joseph JV. Is it worth revisiting laparoscopic three-dimensional visualization? A validated assessment. Urology 2007;70:47-49.

30. Bhayani SB, Andriole GL. Three-dimensional (3D) vision: Does it improve laparoscopic skills? An assessment of a 3D head-mounted visualization system. Rev Urol 2005;7:211214.

31. Schoenthaler M, Schnell D, Wilhelm K, Schlager D, Adams F, Hein S, et al. Stereoscopic (3D) versus monoscopic (2D) laparoscopy: Comparative study of performance using advanced HD optical systems in a surgical simulator model. World J Urol 2016;34:471-477.

32. Honeck P, Wendt-Nordahl G, Rassweiler J, Knoll T. Threedimensional laparoscopic imaging improves surgical performance on standardized ex vivo laparoscopic tasks. J Endourol 2012;26:1085-1088.

33. Sun CC, Chiu AW, Chen KK, Chang LS. Assessment of a three-dimensional operating system with skill tests in a pelvic trainer. Urol Int 2000;64:154-158.
34. Tanaka K, Shigemura K, Ishimura T, Muramaki M, Miyake H, Fujisawa M. Evaluation of a 3D system based on a highquality flat screen and polarized glasses for use by surgical assistants during robotic surgery. Indian J Urol 2014;30:13-16.

35. Badani KK, Bhandari A, Tewari A, Menon M. Comparison of two-dimensional and three-dimensional suturing: Is there a difference in a robotic surgery setting? J Endourol 2005; 19:1212-1215.

36. Hinata $\mathrm{N}$, Iwamoto $\mathrm{H}$, Morizane $\mathrm{S}$, Hikita $\mathrm{K}$, Yao A, Muraoka K, et al. Dry box training with three-dimensional vision for the assistant surgeon in robot-assisted urological surgery. Int J Urol 2013;20:1037-1041.

37. Becker H, Melzer A, Schurr MO, Buess G. 3-D video techniques in endoscopic surgery. Endosc Surg Allied Technol 1993;1:40-46.

38. Ko JK, Li RH, Cheung VY. Two-dimensional versus threedimensional laparoscopy: Evaluation of physicians' performance and preference using a pelvic trainer. J Minim Invasive Gynecol 2015;22:421-427.

39. Gurusamy KS, Sahay S, Davidson BR. Three dimensional versus two dimensional imaging for laparoscopic cholecystectomy. Cochrane Database Syst Rev 2011:CD006882.

40. Bilgen K, Ustun M, Karakahya M, Isik S, Sengul S, Cetinkunar S, et al. Comparison of 3D imaging and 2D imaging for performance time of laparoscopic cholecystectomy. Surg Laparosc Endosc Percutan Tech 2013;23:180-183.

41. Arezzo A, Vettoretto N, Francis NK, Bonino MA, Curtis NJ, Amparore D et al. The use of 3D laparoscopic imaging systems in surgery: EAES consensus development conference 2018. Surg Endosc 2019;33:3251-3274.

42. Dirie NI, Wang Q, Wang S. Two-dimensional versus threedimensional laparoscopic systems in urology: A systematic review and meta-analysis. J Endourol 2018;32:781-790.

43. Tjiam IM, Persoon MC, Hendrikx AJ, et al. Program for laparoscopic urologic skills: A newly developed and validated educational program. J Urol 2012;79:815-820.

44. van Bergen P, Kunert W, Bessell J, Buess GF. Comparative study of two--dimensional and three-dimensional vision systems for minimally invasive surgery. Surg Endosc 1998; 12:948-954.

45. Smith R, Day A, Rockall T, et al. Advanced stereoscopic projection technology significantly improves novice performance of minimally invasive surgical skills. Surg Endosc 2012;26:1522-1527.

46. Li Z, Wang G, Tan J, Sun X, Lin H, Zhu S. Building a framework for ergonomic research on laparoscopic instrument handles. Int J Surg 2016;30:74-82.

47. Hallbeck MS, Lowndes BR, McCrory B, Morrow MM, Kaufman KR, LaGrange CA. Kinematic and ergonomic assessment of laparoendoscopic single-site surgical instruments during simulator training tasks. Appl Ergon 2017;62: 118-130.

48. Sánchez-Margallo FM, Sánchez-Margallo JA. Assessment of postural ergonomics and surgical performance in laparoendoscopic single-site surgery using a handheld robotic device. Surg Innov 2018;25:208-217.

49. Ding D, Jiang H, Nie J, Liu X, Guo SW. Concurrent learning curves of 3-dimensional and robotic-assisted laparoscopic radical hysterectomy for early-stage cervical cancer using 2-dimensional laparoscopic radical hysterectomy as a benchmark: A single surgeon's experience. Med Sci Monit 2019;25:5903-5919.

50. Vettoretto N, Foglia E, Ferrario L, Arezzo A, Cirocchi R, Cocorullo $\mathrm{G}$, et al. Why laparoscopists may opt for 
three-dimensional view: A summary of the full HTA report on $3 \mathrm{D}$ versus $2 \mathrm{D}$ laparoscopy by S.I.C.E. (Società Italiana di Chirurgia Endoscopica e Nuove Tecnologie). Surg Endosc 2018;32:2986-2993.

51. Yun JJ, Kim EY, Ahn AJ, Kim JK, Choi JH, Park JM, et al. A retrospective single-center study comparing clinical outcomes of 3-dimensional and 2-dimensional laparoscopic cholecystectomy in acute cholecystitis. Ann Hepatobiliary Pancreat Surg 2019;23:339-343.

52. Sinha RY, Raje SR, Rao GA. Three-dimensional laparoscopy: Principles and practice. J Minim Access Surg 2017; 13:165-169.

53. Longmore SK, Naik G, Gargiulo GD. Laparoscopic robotic surgery: Current perspective and future directions. Robotics 2020;27;9:42.

54. Falk V, Mintz D, Grunenfelder J, Fann JI, Burdon TA. Influence of three-dimensional vision on surgical telemanipulator performance. Surg Endosc 2001;15:1282-1288.

55. Kastelan Z, Hudolin T, Kulis T, Penezic L, Gidaro S, Bakula $\mathrm{M}$, et al. Extraperitoneal radical prostatectomy with the senhance robotic platform: First 40 cases. Eur Urol 2020 [Epub ahead of print]; DOI: 10.1016/j.eururo.2020 .07 .012 .

56. Thomas BC, Slack M, Hussain M, Barber N, Pradhan A, Dinneen E, et al. Preclinical evaluation of the versius surgical system, a new robot-assisted surgical device for use in minimal access renal and prostate surgery. Eur Urol Focus 2020 [Epub ahead of print]; DOI: 10.1016/j.euf.2020.01.011.

57. Zwart MJW, Jones LR, Balduzzi A, Takagi K, Vanlander $\mathrm{A}$, van den Boezem PB, et al. Added value of 3D-vision during robotic pancreatoduodenectomy anastomoses in biotissue (LAEBOT 3D2D): A randomized controlled cross-over trial. Surg Endosc 2020 [Epub ahead of print]; DOI: $10.1007 / \mathrm{s} 00464-020-07732-\mathrm{z}$.
Address correspondence to: Francisco Miguel Sánchez-Margallo, PhD Jesús Usón Minimally Invasive Surgery Centre

Ctra. N-521, Km. 41.8

10071 Cáceres

Spain

E-mail: msanchez@ccmijesususon.com

$\begin{aligned} & \text { Abbreviations Used } \\ \mathrm{A} & =\text { physician assistant } \\ \mathrm{CI} & =\text { confidence interval } \\ 2 \mathrm{D}= & \text { two-dimensional } \\ 3 \mathrm{D}= & \text { three-dimensional } \\ \mathrm{E} & =\text { experts } \\ \mathrm{EAES} & =\text { European Association of Endoscopic } \\ & \quad \text { Surgery } \\ \mathrm{E}-\mathrm{BLUS}= & \text { European training program in basic } \\ \mathrm{FLS} & =\text { fundamentals of laparoscopic surgery } \\ \mathrm{HD} & =\text { high-definition } \\ \mathrm{I} & =\text { intermediates } \\ \mathrm{IQR} & =\text { interquartile range } \\ \mathrm{MIS} & =\text { minimally invasive surgery } \\ \mathrm{N} & =\text { novices } \\ \mathrm{NA} & =\text { not available } \\ \mathrm{NS} & =\text { not statistically significant } \\ \mathrm{OR} & =\text { operating room } \\ \mathrm{STAI}-6 & =\text { State-Trait Anxiety Inventory for Adults } \\ \mathrm{VUA} & =\text { vesicourethral anastomosis }\end{aligned}$

\section{Appendix}

\section{Appendix A1. Full search strategy}

PubMed: 114 results (24/08/2018)

("Imaging, Three-Dimensional"'[Mesh] OR 3D OR 3-D OR three-dimension* OR 3-dimension*) AND ("Laparoscopy" [Mesh] OR “Laparoscopes"'[Mesh] OR laparosc* OR laparoendosc* OR celioscop* OR "Minimally Invasive Surgical Procedures"'[Mesh:NoExp] OR minimallyinvasive-surg*) AND ("Urinary Tract"'[Mesh] OR "Genitalia, Male"'[Mesh] OR “Male Urogenital Diseases"'[Mesh] OR “Urologic Diseases"'[Mesh] OR "Urologic Surgical Procedures"'[Mesh] OR urol* OR kidney* OR renal OR nephrect* OR nephropex* OR nephroureter* OR ureter OR ureteral* OR ureterect* OR bladder* OR prostate* OR cystectom* OR varicocele* OR cryptorchid* OR retroperitoneal-lymph-node* OR adrenalectomy* OR pye- loplast* OR pelvic-lymph-node*) AND (performance OR ergonom*)

EMBASE: 199 (24/08/2018)

(("three dimensional imaging"/ /exp OR 3d OR "3 d" OR "three dimension*" OR "3 dimension*") AND ("laparoscopy"/exp OR "laparoscope"/exp OR laparosc* OR laparoendosc* OR celioscop* OR "minimally invasive surgery"/ de OR "minimally invasive surg*") AND ("urinary tract"/ exp OR "male genital system"/exp OR "urinary tract disease"/exp OR "male genital system disease"/exp OR "urologic surgery"/exp OR urol* OR kidney* OR renal OR nephrect* OR nephropex* OR nephroureter* OR ureter OR ureteral* OR ureterect* OR bladder* OR prostate* OR cystectom* OR varicocele* OR cryptorchid* OR "retroperitoneal lymph node*" OR adrenalectomy* OR pyeloplast* OR “pelvic lymph node*”)) AND (performance OR ergonom*) 